\title{
ON QUASI-IDEALS OF SEMIRINGS
}

\author{
CHRISTOPH DÖNGES \\ Institut für Mathematik \\ Technische Universität Clausthal \\ Erzstraße 1 \\ DW-3392 Clausthal-Zellerfeld \\ Germany
}

(Received September 28, 1992 and in revised form February 23, 1993)

ABSTRACT. Several statements on quasi-ideals of semirings are given in this paper, where these semirings may have an absorbing element $O$ or not. In Section 2 we characterize regular semirings and regular elements of semi-rings using quasi-ideals (cf. Thms. 2.1, 2.2 and 2.7). In Section 3 we deal with $(O$-)minimal and canonical quasi-ideals. In particular, if the considered semiring $S$ is semiprime or quasi-reflexive, we present criterions which allow to decide easily whether an (O-)minimal quasi-ideal of $S$ is canonical (cf. Thms. 3.4 and 3.8). If $S$ is an arbitrary semiring, we prove that for (O-)minimal left and right ideals $L$ and $R$ of $S$ the product $\langle R L\rangle \subseteq L \cap R$ is either $\{O\}$ or a canonical quasi-ideal of $S$ (Thm. 3.9). Moreover, for each canonical quasi-ideal $Q$ of a semiring $S$ and each element $a \in S, Q a$ is either $\{O\}$ or again a canonical quasi-ideal of $S$ (Thm. 3.11), and the product $\left\langle Q_{1} Q_{2}\right\rangle$ of canonical quasi-ideals $Q_{1}, Q_{2}$ of $S$ is either $\{O\}$ or again a canonical quasi-ideal of $S$ (Thm. 3.12). Corresponding results to those given here for semirings are mostly known as well for rings as for semigroups, but often proved by different methods. All proofs of our paper, however, apply simultaneously to semirings, rings and semigroups (cf. Convention 1.1), and we also formulate our results in a unified way for these three cases. The only exceptions are statements on semirings and semigroups without an absorbing element $O$, which cannot have corresponding statements on rings since each ring has its zero as an absorbing element.

KEY WORDS AND PHRASES. Quasi-ideals, regular elements, regular semirings, $(O$-)minimal quasi-ideals, canonical quasi-ideals.

1992 AMS SUBJECT CLASSIFICATION CODES. 16 Y 60, 12 K 10, 16 D 80, 20 M 12.

\section{PRELIMINARIES.}

An algebra $S=(S,+, \cdot)$ is called a semiring, iff $(S,+)$ and $(S, \cdot)$ are arbitrary semigroups connected by ring-like distributivity. If there is an element $o \in S[e \in S]$ satisfying $o+a=a+o=a$ $[e a=a e=a$ ] for all $a \in S$, it is called the zero [the identity] of $S$. If there is an element $O \in S$ satisfying $O a=a O=O$ for all $a \in S$, it is called the absorbing element of $S$. Note that there are semirings with a zero $o$ satisfying $o=e$ or $o o \neq o$, whereas $O+O=O$ trivially holds for each semiring with an absorbing element $O$. A semiring with elements $O$ and $O$ which coincide is said to have an absorbing zero $o=O$; among others, each ring is an example for such a semiring. Let $\mathbb{I N}$ be the set of positive integers and $\emptyset \neq X, Y \subseteq S$. We introduce the notion 


$$
\langle X\rangle=\left\{\sum_{\imath=1}^{n} x_{\imath} \mid x_{\imath} \in X, n \in \mathbb{N}\right\}
$$

for the subsemigroup of $(S,+)$ generated by $X$. Deviating from the usual notion in ring theory, we define $X Y$ for semirings (and hence for rings) $(S,+, \cdot)$ in the same way as for semigroups $(S, \cdot)$ by

$$
X Y=\{x y \mid x \in X, y \in Y\}
$$

The product of subsets mostly used in ring theory can then be expressed by $\langle X Y\rangle$ according to

$$
\langle X Y\rangle=\left\{\sum_{i=1}^{n} x_{i} y_{i} \mid x_{i} \in X, y_{i} \in Y, n \in \mathbb{N}\right\}
$$

Clearly, we write e.g. $x Y$ and $\langle x Y\rangle$ instead of $\{x\} Y$ and $\langle\{x\} Y\rangle$. We have to use both notions (1.2) and (1.3) extensively, including the following equations concerning (1.3) which are easily checked for all $\emptyset \neq X, Y, Z \subseteq S$ :

$$
\begin{aligned}
& \langle X \cup Y\rangle=\langle\langle X\rangle \cup\langle Y\rangle\rangle \\
& \langle X Y\rangle=\langle\langle X\rangle Y\rangle=\langle X\langle Y\rangle\rangle \\
& \langle X Y Z\rangle=\langle\langle X Y\rangle Z\rangle=\langle X\langle Y Z\rangle\rangle=\langle\langle X\rangle\langle Y\rangle\langle Z\rangle\rangle .
\end{aligned}
$$

Whereas in general only $X Y \subseteq\langle X Y\rangle$ is true, clearly $x Y=\langle x Y\rangle$ and $Y x=\langle Y x\rangle$ hold for all $x \in S$ and all $Y \subseteq S$ satisfying $Y=\langle Y\rangle$.

Our main interest is with quasi-ideals of semirings, and we also need left, right and two-sided ideals. Since these concepts differ for semirings and rings, we recall them in a way pointing out this difference: A subset $\emptyset \neq L \subseteq S$ of a (semi)ring $(S,+, \cdot)$ is called a left ideal of $(S,+, \cdot)$ iff $L$ is a sub(semi)group of $(S,+)$ satisfying $S L \subseteq L$. The latter states that $L$ is a left ideal of $(S, \cdot)$, and for an additive sub(semi)group $L$ of a (semi)ring $S$, clearly, $S L \subseteq L$ and $\langle S L\rangle \subseteq L$ are equivalent. Right ideals and two-sided ideals of $(S,+, \cdot)$ are defined analogously. Due to [3], §12, a subset $\emptyset \neq Q \subseteq S$ of a (semi)ring $(S,+, \cdot)$ is called a quasi-ideal of $(S,+, \cdot)$, iff $Q$ is a sub(semi)group of $(S,+)$ satisfying $\langle S Q\rangle \cap\langle Q S\rangle \subseteq Q$. In this case the condition $S Q \cap Q S \subseteq Q$, stating that $Q$ is a quasi-ideal of the semigroup $(S, \cdot)$, leads to the concept of a weak quasi-ideal of the (semi)ring $(S,+, \cdot)$, and such a weak quasi-ideal need not be a quasi-ideal (cf. [8], §2).

In general, if $(S,+, \cdot)$ is a ring, one may consider left, right, two-sided and quasi-ideals in the ring-theoretical as well as in the semiring-theoretical meaning. Since each subgroup is all the more a subsemigroup, each ring-theoretical ideal is a semiring-theoretical one. The converse holds e.g. for rings with an identity, but not in general.

As already mentioned in the abstract, most of our results on semirings correspond to already known statements on semigroups and on rings. Moreover, even in cases where those statements have been proved for semigroups and for rings by completely different ideas, our proof for the corresponding statement on semirings will apply also to semigroups and rings. So we want to formulate our considerations in such a way, that they can be read for semirings as well as for semigroups and rings (the latter of course for rings considered as rings and not as a special kind of semirings). For this reason, we use the following 
CONVENTION 1.1. a) If $S=(S,+, \cdot)$ is a ring, the terms left, right, two-sided or quasi-ideal of $S$ are used in the ring-theoretical meaning.

b) The notions $\langle X\rangle, X Y$ and $\langle X Y\rangle$ have always the same meaning according to (1.1). (1.2) and (1.3), regardless whether $S=(S,+, \cdot)$ is a semiring or a ring. (This will cause no problems, since in the ring-case $\langle X\rangle$ is a subgroup of $(S,+)$, provided that $-X \subseteq X$ holds.)

c) In the case that $S=(S, \cdot)$ is a semigroup, one has to pay no attention to the brackets \langle\rangle .

In this context we remark, that each of our results on semirings provides automatically the corresponding one on semigroups. The reason is that any semigroup $(S, \cdot)$ determines a semiring $(S,+, \cdot)$ with respect to the left absorbing addition on $S$, defined by $a+b=a$ for all $a, b \in S$, such that each left, right, two-sided or quasi-ideal of $(S, \cdot)$ is an ideal of the same kind of $(S,+, \cdot)$, and conversely.

The elementary properties given in the following two Lemmata are well known (cf. e.g. [3], §1-2 for rings and semigroups and [8], $\S 1$ for semirings).

LEMMA 1.2. Let $S$ be a semiring, a ring or a semigroup.

a) Each one- or two-sided ideal of $S$ is a quasi-ideal of $S$.

b) The intersection of any system of quasi-ideals of $S$ is either empty or a quasi-ideal of $S$.

c) If $L$ is a left and $R$ a right ideal of $S$, then $R L \subseteq\langle R L\rangle \subseteq L \cap R$ holds and the intersection $Q=L \cap R$ is a quasi-ideal of $S$.

d) For each $\emptyset \neq X \subseteq S,\langle S X\rangle$ is a left ideal, $\langle X S\rangle$ a right ideal, $\langle S X S\rangle$ an ideal and $\langle S X\rangle \cap\langle X S\rangle$ a quasi-ideal of $S$.

e) Each quasi-ideal of a semiring, a ring or a semigroup $S$ is a subsemiring, a subring or a subsemigroup of $S$ respectively.

LEMMA 1.3. Let $S$ be a semiring, a ring or a semigroup, $L$ a left and $R$ a right ideal of $S$ and consider elements $e=e^{2}$ and $f=f^{2}$ of $S$. Then we have

$$
e L=L \cap e S, \quad R e=S e \cap R \text { and } S f \cap e S=e S f
$$

and all three subsets are quasi-ideals of $S$.

For each $\emptyset \neq X \subseteq S$ we denote by $(X)_{l},(X)_{r},(X)_{t}$ and $(X)_{q}$ the left, ight, two-sided and the quasi-ideal of $S$ generated by $X$. We call them principle if they can be generated by one element $x$ and write then e.g. $(x)_{q}$ instead of $(\{x\})_{q}$. The following lemma can be checked in a straightforward manner.

LEMMA 1.4. Let $S$ be a semiring, a ring or a semigroup.

a) For each $\emptyset \neq X \subseteq S$, additionally satisfying $-X \subseteq X$ in the case that $S$ is a ring, we have

$$
\begin{aligned}
& (X)_{l}=\langle X \cup S X\rangle,(X)_{r}=\langle X \cup X S\rangle, \\
& (X)_{t}=\langle X \cup S X \cup X S \cup S X S\rangle \text { and }(X)_{q}=\langle X \cup(\langle S X\rangle \cap\langle X S\rangle)\rangle .
\end{aligned}
$$

b) For the principle left, right and two-sided ideals generated by $s \in S$ we have $\left\langle S(s)_{l}\right\rangle=S s$, $\left\langle(s)_{r} S\right\rangle=s S$ and $\left\langle S(s)_{t} S\right\rangle=\langle S s S\rangle$.

Further, a quasi-ideal $Q$ of $S$ is said to have the intersection property, iff $Q=L \cap R$ holds for suitable left and right ideals $L$ and $R$ of $S$ which clearly implies $Q=(Q)_{l} \cap(Q)_{r}$. Whereas each quasi-ideal of a semigroup $(S, \cdot)$ has the intersection property ([3], Prop. 2.6), an example due to Clifford (published in [3], Expl. 2.1) shows that there are rings which contain quasi-ideals without 
the intersection property. Examples given by Weinert ([8], Prop. 5.2) show that there are even $O$-minimal (cf. Section 3) quasi-ideals of a semiring $(S,+, \cdot)$ with absorbing element $O$, which do not have the intersection property. Finally, a bi-ideal $B$ of the (semi)ring $(S,+, \cdot)$ is defined as a sub(semi)ring of $(S,+, \cdot)$ satisfying $B S B \subseteq B$ and hence $\langle B S B\rangle \subseteq B$. Note that $B S B \subseteq B$ states that the subsemigroup $B$ of $(S, \cdot)$ is a bi-ideal of the semigroup $(S, \cdot)$. The following lemma is again easy to check:

LEMMA 1.5. Let $S$ be a semiring, a ring or a semigroup and $T$ a two-sided ideal of $S$. Then each quasi-ideal $Q$ of $T$ is a bi-ideal of $S$. Especially each quasi-ideal $Q$ of $S$ is also a bi-ideal of $S$; hence $Q$ satisfies $\langle Q S Q\rangle \subseteq Q$.

\section{QUASI-IDEALS AND REGULARITY}

Let $S$ be a semiring, a ring or a semigroup. Then an element $s \in S$ is called regular in $S$ iff $s \in s S s$ holds, and $S$ is called regular iff each element of $S$ is regular in S. Note that an element $s$ of a semiring, a ring or a semigroup $S$ is regular iff one of the following statements holds:

(2.1) There is an element $x \in S$ satisfying $x s=e=e^{2}, \quad s=s e$.

$\left(2.1^{\prime}\right)$ There is an element $y \in S$ satisfying $s y=f=f^{2}, \quad s=f s$.

(2.2) There is an element $e=e^{2} \in S$ satisfying $(s)_{l}=S e=S s$.

$\left(2.2^{\prime}\right)$ There is an element $f=f^{2} \in S$ satisfying $(s)_{r}=f S=s S$.

Restricted to the special cases that $S$ is a ring or a semigroup, most of the results of this section can be found in [3], §9.

THEOREM 2.1. Let $S$ be a semiring, a ring or a semigroup. Then the following conditions are equivalent:

(1) $S$ is regular.

(2) Each left ideal $L$ and each right ideal $R$ of $S$ satisfy $\langle R L\rangle=L \cap R$ (which in fact implies $R L=\langle R L\rangle=L \cap R)$.

(3) Each left ideal $L$ and each right ideal $R$ of $S$ satisfy

a) $\left\langle L^{2}\right\rangle=L$,

b) $\left\langle R^{2}\right\rangle=R$ and

c) $\langle R L\rangle$ is a quasi-ideal of $S$.

(4) The set $\mathcal{Q}$ of all quasi-ideals of $S$ is a regular semigroup with respect to the "product" $\left\langle Q_{1} Q_{2}\right\rangle$.

(5) Each quasi-ideal $Q$ of $S$ satisfies $Q=\langle Q S Q\rangle$.

Moreover, the statements 3a) and 3b) imply that each quasi-ideal $Q$ of $S$ has the intersection property since it satisfies $Q=\langle S Q\rangle \cap\langle Q S\rangle$.

PROOF. At first we prove the last statement. We apply 3a) to the left ideal $(Q)_{l}=\langle Q \cup S Q\rangle$ of $S$ generated by a quasi-ideal $Q$ of $S$ and obtain $Q \subseteq\langle Q \cup S Q\rangle=\left\langle\langle Q \cup S Q\rangle^{2}\right\rangle \subseteq\langle S Q\rangle$, where the last inclusion is obvious. Similarly we get $Q \subseteq\langle Q \cup Q S\rangle=\left\langle\langle Q \cup Q S\rangle^{2}\right\rangle \subseteq\langle Q S\rangle$ and therefore $Q \subseteq\langle S Q\rangle \cap\langle Q S\rangle \subseteq Q$.

(1) $\Rightarrow$ (2): The inclusion $R L \subseteq\langle R L\rangle \subseteq L \cap R$ holds by Lemma $1.2 \mathrm{c})$. On the other hand, for each $d \in L \cap R$ there exists an $x \in S$ such that $d=d x d$ since $S$ is regular. Now $d \in R$ and $x d \in S L \subseteq L$ imply $d \in R L$.

$(2) \Rightarrow(3):$ For a), let $L$ be a left ideal of $S$ and $(L)_{r}=\langle L \cup L S\rangle$ the right (in fact two-sided) ideal of $S$ generated by $L$. Then (2) implies 


$$
L=L \cap(L)_{r}=\left\langle(L)_{r} L\right\rangle=\langle\langle L \cup L S\rangle L\rangle \subseteq\langle L L \cup L S L\rangle=\langle L L\rangle \subseteq L .
$$

The statement $\mathrm{b}$ ) can be proved dually and $\langle R L\rangle=L \cap R$ is a quasi-ideal by Lemma $1.2 \mathrm{c}$ ).

(3) $\Rightarrow(4)$ : At first the multiplication (1.3) is associative by (1.6). Let $Q_{1}$ and $Q_{2}$ be quasi-idcals of $\mathrm{S}$. Then $L=\left\langle S Q_{1} Q_{2}\right\rangle$ and $R=\left\langle Q_{1} Q_{2} S\right\rangle$ are left and right ideals of $S$, hence 3a) and 3b) imply $S=\left\langle S^{2}\right\rangle$ and

$$
\begin{aligned}
& \left\langle S Q_{1} Q_{2}\right\rangle=\left\langle\left\langle S Q_{1} Q_{2}\right\rangle\left\langle S S Q_{1} Q_{2}\right\rangle\right\rangle=\left\langle S\left\langle Q_{1} Q_{2} S\right\rangle\left\langle S Q_{1} Q_{2}\right\rangle\right\rangle \\
& \left\langle Q_{1} Q_{2} S\right\rangle=\left\langle\left\langle Q_{1} Q_{2} S S\right\rangle\left\langle Q_{1} Q_{2} S\right\rangle\right\rangle=\left\langle\left\langle Q_{1} Q_{2} S\right\rangle\left\langle S Q_{1} Q_{2}\right\rangle S\right\rangle
\end{aligned}
$$

Moreover, $\langle R L\rangle$. is a quasi-ideal of $S$ by 3c) and satisfies $\langle S R L\rangle \cap\langle R L S\rangle=\langle R L\rangle$, due to the last statement of the theorem. So we obtain

$$
\begin{aligned}
\left\langle S Q_{1} Q_{2}\right\rangle \cap\left\langle Q_{1} Q_{2} S\right\rangle & =\left\langle S\left\langle Q_{1} Q_{2} S\right\rangle\left\langle S Q_{1} Q_{2}\right\rangle\right\rangle \cap\left\langle\left\langle Q_{1} Q_{2} S\right\rangle\left\langle S Q_{1} Q_{2}\right\rangle S\right\rangle \\
& =\langle S R L\rangle \cap\langle R L S\rangle=\langle R L\rangle=\left\langle\left\langle Q_{1} Q_{2} S\right\rangle\left\langle S Q_{1} Q_{2}\right\rangle\right\rangle \\
& \subseteq\left\langle Q_{1} Q_{2} S Q_{2}\right\rangle \subseteq\left\langle Q_{1} Q_{2}\right\rangle
\end{aligned}
$$

the last step by Lemma 1.5. Hence $\left\langle Q_{1} Q_{2}\right\rangle$ is again a quasi-ideal of $S$, i.e. $\mathcal{Q}$ is a semigroup and it remains to show that it is regular. Each quasi-ideal $Q \in \mathcal{Q}$ satisfies $Q=\langle S Q\rangle \cap\langle Q S\rangle$, again by the last statement of the theorem, and in a similar way as above we can conclude

Hence $Q$ is regular in $\mathcal{Q}$.

$$
\begin{aligned}
Q & =\langle S Q\rangle \cap\langle Q S\rangle=\langle\langle S Q\rangle\langle S S Q\rangle\rangle \cap\langle\langle Q S S\rangle\langle Q S\rangle\rangle \\
& =\langle S\langle Q S\rangle\langle S Q\rangle\rangle \cap\langle\langle Q S\rangle\langle S Q\rangle S\rangle=\langle\langle Q S\rangle\langle S Q\rangle\rangle=\langle Q S Q\rangle .
\end{aligned}
$$

$(4) \Rightarrow(5)$ : For each quasi-ideal $Q$ of $S$ there exists a quasi-ideal $X$ of $S$ such that $Q=\langle Q X Q\rangle \subseteq$ $\langle Q S Q\rangle \subseteq\langle S Q\rangle \cap\langle Q S\rangle \subseteq Q$, hence $Q=\langle Q S Q\rangle$.

$(5) \Rightarrow(1)$ : For each element $s \in S$ the intersection $(s)_{l} \cap(s)_{r}$ is a quasi-ideal of $S$ by Lemma $\left.1.2 \mathrm{c}\right)$. Using (5) and Lemma $1.4 \mathrm{~b}$ ) we conclude

$$
\begin{aligned}
s \in(s)_{l} \cap(s)_{r} & =\left\langle\left((s)_{l} \cap(s)_{r}\right) S\left((s)_{l} \cap(s)_{r}\right)\right\rangle \\
& \subseteq\left\langle(s)_{r} S(s)_{l}\right\rangle=\left\langle s S(s)_{l}\right\rangle=\langle s S s\rangle=s S s .
\end{aligned}
$$

Hence each $s \in S$ is regular in $S$.

For regular elements of a semiring, a ring or a semigroup $\mathrm{S}$ there is the following analogue of Theorem 2.1:

THEOREM 2.2. The following statements about an element $s$ of a semiring, a ring or a semigroup $S$ are equivalent:

(1) The element $s$ is regular in $S$.

(2) The principle left ideal $(s)_{l}$ and the principle right ideal $(s)_{r}$ of $S$ satisfy

$$
\left\langle(s)_{r}(s)_{l}\right\rangle=(s)_{l} \cap(s)_{r} .
$$

(3) The principle left ideal $(s)_{l}$ and the principle right ideal $(s)_{r}$ of $S$ satisfy

a) $\left\langle(s)_{l}^{2}\right\rangle=(s)_{l}$,

b) $\left\langle(s)_{r}^{2}\right\rangle=(s)_{r}$ and

c) $\left\langle(s)_{r}(s)_{l}\right\rangle$ is a quasi-ideal of $S$.

(4) The principle quasi-ideal $(s)_{q}$ of $S$ satisfies $(s)_{q}=\left\langle(s)_{q} S(s)_{q}\right\rangle$. 
PROOF. (1) $\Rightarrow(3)$ : For a) let $s \in S$ be regular in $S$. By (2.2) and $\left(2.2^{\prime}\right)$ we have $(s)_{l}=S e$ for some $e=e^{2} \in S$ and $(s)_{r}=f S$ for some $f=f^{2} \in S$. From $(s)_{l}=S e \subseteq\langle S e S e\rangle=\left\langle(s)_{l}^{2}\right\rangle$ we obtain $\left\langle(s)_{l}^{2}\right\rangle=(s)_{l}$ since $(s)_{l}^{2} \subseteq(s)_{l}$ is clear. The statement b) can be proved dually, and $\left\langle(s)_{r}(s)_{l}\right\rangle=\langle f S S e\rangle$ is a quasi-ideal of $S$ by Lemma 1.3 since $\langle f S S\rangle$ is a right ideal of $S$.

$(3) \Rightarrow(2)$ : From 3a) one obtains $(s)_{l}=\left\langle(s)_{l}^{2}\right\rangle \subseteq\left\langle S(s)_{l}\right\rangle=S s$ by Lemma $\left.1.4 \mathrm{~b}\right)$, that is $(s)_{l}=S s$. Similarly $3 \mathrm{~b}$ ) yields $(s)_{r}=s S$. Using again $\left.3 \mathrm{a}\right)$ and $\left.3 \mathrm{~b}\right)$ one obtains $(s)_{l} \cap(s)_{r}=S s \cap s S=$ $\left\langle(S s)^{3}\right\rangle \cap\left\langle(s S)^{3}\right\rangle$. Now $\left\langle(s)_{r}(s)_{l}\right\rangle=\langle s S S s\rangle$ is by 3c) a quasi-ideal of $S$. From this it follows $\langle S s S S s\rangle \cap\langle s S S s S\rangle \subseteq\langle s S S s\rangle=\left\langle(s)_{r}(s)_{l}\right\rangle$ and therefore

$$
(s)_{l} \cap(s)_{r}=\left\langle(S s)^{3}\right\rangle \cap\left\langle(s S)^{3}\right\rangle \subseteq\langle S s S S s\rangle \cap\langle s S S s S\rangle \subseteq\left\langle(s)_{r}(s)_{l}\right\rangle .
$$

This proves (2), since the other inclusion is clear.

$(2) \Rightarrow(4)$ : Obviously we have $\left\langle(s)_{q} S(s)_{q}\right\rangle \subseteq\left\langle S(s)_{q}\right\rangle \cap\left\langle(s)_{q} S\right\rangle \subseteq(s)_{q}$. From (2) and Lemma 1.4 b) it follows $s \in(s)_{l} \cap(s)_{r}=\left\langle(s)_{r}(s)_{l}\right\rangle \subseteq\left\langle S(s)_{l}\right\rangle=S s$, hence $(s)_{l}=S s$ and similarly $(s)_{r}=s S$. So we obtain $(s)_{q} \subseteq(s)_{l} \cap(s)_{r}=\left\langle(s)_{r}(s)_{l}\right\rangle=\langle s S S s\rangle \subseteq\left\langle(s)_{q} S(s)_{q}\right\rangle$.

(4) $\Rightarrow(1)$ : We have $s \in(s)_{q}=\left\langle(s)_{q} S(s)_{q}\right\rangle \subseteq\left\langle(s)_{r} S(s)_{l}\right\rangle=\left\langle s S(s)_{l}\right\rangle=s S s$, the last stcps again by Lemma $1.4 \mathrm{~b})$, so $s$ is regular in $S$.

By the aid of Thm. 2.1, we obtain further properties of quasi-ideals in regular semirings, rings and semigroups. In this context we need the following

LEMMA 2.3. Each two-sided ideal $T$ of a regular semiring, ring or semigroup $S$ is a regular subsemiring, subring or subsemigroup of $S$.

PROOF. Each element $s \in T \subseteq S$ is regular in $S$, so there is an $x \in S$ such that $s=s x s=$ $s x(s x s)=s(x s x) s$. Since $x s x$ is an element of $T, s$ is regular in $T$, too.

THEOREM 2.4. Let $S$ be a regular semiring, ring or semigroup. Then the following statements are true:

a) Each quasi-ideal $Q$ of $S$ satisfies

$$
Q=L \cap R=\langle R L\rangle \quad \text { with } L=(Q)_{l}=\langle S Q\rangle \text { and } R=(Q)_{r}=\langle Q S\rangle .
$$

b) Each quasi-ideal $Q$ of $S$ satisfies $\left\langle Q^{2}\right\rangle=\left\langle Q^{3}\right\rangle$.

c) Each bi-ideal $B$ of $S$ is a quasi-ideal of $S$.

d) Each bi-ideal $B$ of a two-sided ideal $T$ of $S$ is a quasi-ideal of $S$.

With respect to b) we note, that the regularity of $S$ does not imply $Q=\left\langle Q^{2}\right\rangle$. Moreover, the statement c) does not imply that $S$ is regular.

PROOF. a) By Theorem 2.1 each quasi-ideal $Q$ of $S$ has the intersection property $Q=$ $\langle S Q\rangle \cap\langle Q S\rangle=(Q)_{l} \cap(Q)_{r}=L \cap R$ and condition (2) of Theorem 2.1 implies $Q=L \cap R=\langle R L\rangle$.

b) For each quasi-ideal $Q$ of $S$ it follows by Theorem 2.1 (4), that $\left\langle Q^{2}\right\rangle$ is also a quasi-ideal of $S$ and that there is a quasi-ideal $X$ of $S$ such that $\left\langle Q^{2}\right\rangle=\left\langle Q^{2} X Q^{2}\right\rangle \subseteq\left\langle Q^{2} S Q^{2}\right\rangle=\langle Q(Q S Q) Q\rangle \subseteq\left\langle Q^{3}\right\rangle$, which yields $\left\langle Q^{2}\right\rangle=\left\langle Q^{3}\right\rangle$ since $\left\langle Q^{3}\right\rangle \subseteq\left\langle Q^{2}\right\rangle$ is clear.

c) Since $\langle S B\rangle$ is a left ideal and $\langle B S\rangle$ is a right ideal of $S$, Theorem 2.1 (2) implies $\langle S B\rangle \cap\langle B S\rangle=$ $\langle B S S B\rangle \subseteq\langle B S B\rangle \subseteq B$, so $B$ is a quasi-ideal of $S$.

d) By Lemma 2.3 the two-sided ideal $T$ of $S$ is a regular sub(semi)ring or subsemigroup of $S$. So by part c) the bi-ideal $B$ of $T$ is a quasi-ideal of $T$, hence by Lemma 1.5 a bi-ideal of $S$ and again by part c) a quasi-ideal of $S$.

The last statements are proved by the following examples: 
EXAMPLE 2.5. Let $S=M_{2,2}(K)$ be the set of the $(2,2)$-matrices over $K=\boldsymbol{Z} /(2)$. With the usual multiplication (and addition) we consider $S$ as a semigroup, as a semiring and as a ring. Then, in all three cases, $S$ is regular and

$$
Q=\left\{O=\left(\begin{array}{ll}
0 & 0 \\
0 & 0
\end{array}\right),\left(\begin{array}{ll}
0 & 1 \\
0 & 0
\end{array}\right)\right\}
$$

is a quasi-ideal of $S$ (in fact a canonical and hence an O-minimal one, cf. Section 3). Obriously, one has $Q \neq\left\langle Q^{2}\right\rangle=\{O\}$.

EXAMPLE 2.6. In the semiring $S=\mathbb{N}$ with the usual (commutative) operations cach subsemiring of $S$ is an ideal and hence a quasi-ideal of $S$. Thus the same holds for each bi-icleal of $S$. However, only the elcment 1 is regular in $S$. The corresponding statements hold for the semigroup $S=\mathbb{N}$ with respect to multiplication and for the ring $S=\boldsymbol{Z}$ of integers.

Whereas the regularity of $S$ does not imply that $\left\langle Q^{2}\right\rangle=Q$ holds for each quasi-ideal $Q$ of $S$ as just stated, the converse implication is true. Hence the next theorem gives equivalent characterizations for special classes of regular semirings, rings or semigroups:

THEOREM 2.7. The following conditions on a semiring, ring or semigroup $S$ are equivalent: (1) Each left ideal $L$ and each right ideal $R$ of $S$ satisfy

$$
\langle R L\rangle=L \cap R \subseteq\langle L R\rangle
$$

(2) The set $\mathcal{Q}$ of all quasi-ideals of $S$ is an idempotent semigroup with respect to the "product" $\left\langle Q_{1} Q_{2}\right\rangle$.

(3) Each quasi-ideal $Q$ of $S$ satisfies $Q=\left\langle Q^{2}\right\rangle$.

PROOF. To prove (1) $\Rightarrow(2)$ we state at first, that the equality of condition (1) is just the condition (2) of Thm. 2.1, which yields that $S$ is regular and that the set $\mathcal{Q}$ of all quasi-ideals of $S$ is a regular semigroup with respect to the product $\left\langle Q_{1} Q_{2}\right\rangle$. So we only have to show that it is in fact idempotent. We have $Q=\langle Q S Q\rangle$ for each quasi-ideal $Q$ of $S$ by condition (5) of Thm. 2.1 and of course $\left\langle S^{2}\right\rangle=S$ for the left ideal $S$ of $S$ by condition (3) of Thm. 2.1. Combining this we can conclude $Q=\langle Q S Q\rangle=\langle(Q S Q)(S S)(Q S Q)\rangle=\langle Q S\langle Q S S Q\rangle S Q\rangle \subseteq\langle Q S\langle S Q Q S\rangle S Q\rangle=$ $\langle(Q S Q)(Q S Q)\rangle=\left\langle Q^{2}\right\rangle$, where the inclusion follows from (1) since $\langle S Q\rangle$ and $\langle Q S\rangle$ are left and right ideals of $S$, respectively. This yields $Q=\left\langle Q^{2}\right\rangle$ since the other inclusion is clear, hence we proved (2). The implication (2) $\Rightarrow(3)$ is only a restriction. To prove (3) $\Rightarrow(1)$ we use that by Lemma $1.2 \mathrm{c}$ ) for each left ideal $L$ and each right ideal $R$ of $S$ the inclusion $\langle R L\rangle \subseteq L \cap R$ holds and the intersection $L \cap R$ is a quasi-ideal of $S$. So (3) implies $L \cap R=\left\langle(L \cap R)^{2}\right\rangle$ which yields $L \cap R \subseteq\langle R L\rangle$ as well as $L \cap R \subseteq\langle L R\rangle$.

\section{3. (0-)MINIMAL AND CANONICAL QUASI-IDEALS}

Whereas a ring always has an absorbing element $O$ (namely its zero), the considered semigroups and semirings may have such an element or not. In particular, the absorbing element $O$ of a semiring $S$ need not be a zero of $S$, but from $O+O=O$ it follows that $\{O\}$ is a two-sided ideal of $S$ (and hence a quasi-ideal of $S$, cf. Lemma 1.2 a)) also in this case.

For a semiring, a ring or a semigroup $S$ and each $\emptyset \neq X \subseteq S$ we introduce

$$
X^{\prime}= \begin{cases}X \backslash\{O\} & \text { if } S \text { has an absorbing element } O \\ X & \text { otherwise }\end{cases}
$$


Let $Q$ be a quasi-ideal of a semiring, a ring or a semigroup $S$. Then $Q$ is called a minimal quasiidcal of $S$, iff $S^{\prime}=S$ holds and $Q$ does not contain a quasi-ideal $Q_{1}$ of $S$ properly. Moreover, $Q$ is called $O-$ minimal , iff $S^{\prime} \subset S$ and $\{O\} \subset Q$ are satisfied and $Q$ does not contain a quasi-ideal $Q_{1} \neq\{O\}$ of $S$ properly. For convenience, we use $(O$-)minimal when dealing with both cases simultaneously. Analogously one defines $(O-)$ minimal left-, right- and two-sided ideals of $S$. Note that according to this definition a ring $S$ can only have $O$-minimal quasi-ideals and no minimal ones, which deviates again from the usual ring-theoretical terminology.

A quasi-ideal $Q \neq\{O\}$ of a semiring, a ring or a semigroup $S$ is called canonical iff

(3.2) $\quad L$ and $R$ can be chosen $(O$-)minimal.

We note that (3.1) implies $Q=(Q)_{l} \cap(Q)_{r}$ and (3.2) means, that $(Q)_{l}$ and $(Q)_{r}$ are $(O$-)minimal (which conversely implies that $Q$ is canonical).

In the following two theorems we collect known results concerning the relation between the properties that a quasi-ideal $Q$ is $(O$-)minimal or canonical. At first we assume that $Q$ is canonical and ask if it is also $(O-)$ minimal:

THEOREM 3.1. Let $S$ be a semiring, a ring or a semigroup. Then, for each $(O$-)minimal left ideal $L$ and (O-)minimal right ideal $R$ the intersection $Q=L \cap R$ is an (O-)minimal quasi-ideal of $S$ or satisfies $Q=\{O\}$.

Consequently, each canonical quasi-ideal $Q$ of $S$ is an (O-)minimal one.

This has been proved for semirings in [8], Prop. 4.3, for semigroups without an absorbing element in [3], Thm. 5.1 and for semigroups with such an element $O$ and for rings in [3], Thm. 6.1.

Secondly, we assume that a quasi-ideal $Q$ is $(O$-)minimal and ask whether it is also canonical or satisfies at least (3.1). Here we have to distinguish several cases:

THEOREM 3.2. a) Let $S$ be a semiring or a semigroup without an absorbing element. Then each minimal quasi-ideal is also a canonical quasi-ideal of $S$.

b) Let $S$ be a semigroup with absorbing element $O$ and $Q$ an $O$-minimal quasi-ideal of $S$. Then $Q$ has at least the intersection property (3.1).

c) Let $S$ be a semiring or a semigroup with absorbing element $O$ or a ring and $Q$ an $O$-minimal quasi-ideal of $S$. Then either $Q^{2}$ equals $\{O\}$ or $\left(Q^{\prime}, \cdot\right)$ is a subgroup of $(S, \cdot)$. In the latter case, $Q$ satisfies (3.1) since $S x \cap x S=Q$ holds for all $x \in Q^{\prime}$. In particular $Q=S e \cap e S=e S e$ holds with the identity $e$ of $\left(Q^{\prime}, \cdot\right)$. However, $Q$ need not be canonical, since there are examples such that even the left ideal $S e$ and the right ideal $e S$ of $S$ generated by $e \in Q$ are not $O$-minimal.

We note that statement a) is proved for semirings in [8], Satz 4.7 and for semigroups in [3], Thm. 5.1. Statement b) is part of Prop. 2.6 in [3]. The positive statements of $c$ ) can be obtained for semirings by Satz 4.6 and Satz 4.5 of [8] and for semigroups and rings by Thm. 6.3, Cor.6.4 and Thm. 6.5 of [3]. Finally, Example 2.2 in [1] deals with the semigroup $S=\{O, e, a, b\}$ for which all products are $O$ excepted $e^{2}=e, e a=a$ and $b e=b$. It contains the $O$-minimal quasi-ideal $Q=e S e=\{O, e\}$, but neither the left ideal $S e$ nor the right ideal $e S$ are $O$-minimal. We show that examples of this kind yield corresponding ones for rings and semirings:

EXAMPLE 3.3. Let $S$ be a semigroup with above properties, satisfying in particular that the $O$-minimal quasi-ideal $Q$ consists of the two elements $e^{2}=e$ and $O$. Let $A$ be the algebra over a field $F$ with $O$ as its zero and the elements of $S^{\prime}=S \backslash\{O\}$ as a basis. Then $e A e=F e$ is obviously an $O$-minimal quasi-ideal of $A$, but neither the left ideal $A e \supseteq S e$ nor the right ideal $e A \supseteq e S$ are 
$O$-minimal. If one replaces $F$ by a commutative semifield $H$ with an absorbing zcro $O$ (c.g. by the semificld of non-negative rational numbers), the same holds for the resulting scmiring $A$. the semialgebra $A$ over $H$ with $S^{\prime}$ as a basis, cf. [10], Def. 4.1.

In vicw of Theorem 3.2 one may ask for conditions on $S$ which are necessary and sufficient such that each $O$-minimal quasi-ideal of $S$ is also a canonical one. To our knowledge, no conditions of this kind are known. In the following we obtain complete answers in the cases that $S$ is scmiprime or quasi-reflexive (cf. Thm. 3.4 and Thm. 3.8). Both concepts are well known for a semigroup $S$ with an absorbing element $O$ and for a ring $S$. For a semiring $S$ with an absorbing element $O$ we define them in the same way, although $O$ need not be the zero of $S$.

Let $S$ be a semiring or semigroup with absorbing element $O$ or a ring. Then $S$ is called semiprime iff one of the following three conditions (which are easily checked to be equivalent) is satisfied:

(i) if $T$ is a two-sided ideal of $S$ such that $\left\langle T^{2}\right\rangle \subseteq\{O\}$, then $T \subseteq\{O\}$;

(ii) if $L$ is a left ideal of $S$ such that $\left\langle L^{2}\right\rangle \subseteq\{O\}$, then $L \subseteq\{O\}$;

(iii) if $R$ is a right ideal of $S$ such that $\left\langle R^{2}\right\rangle \subseteq\{O\}$, then $R \subseteq\{O\}$.

We recall in this context that $\{O\}=(O)_{t}$ is a two-sided ideal of $S$ in each of the three cases we consider, which yields that the brackets \langle\rangle in (i), (ii) and (iii) are superfluous.

In the case of semiprime rings and semiprime semigroups, the following theorem is essentially Thm. 7.2 in [3].

THEOREM 3.4. Let $S$ be a semiring or semigroup with absorbing element $O$ or a ring such that $S$ is semiprime. Then each $O$-minimal quasi-ideal $Q$ of $S$ is also a canonical quasi-ideal of $S$.

PROOF. At first we state, that $\langle S Q\rangle \neq\{O\}$ holds since otherwise $Q \neq\{O\}$ would be a left ideal of $S$ and satisfy $Q^{2}=\{O\}$, contradicting that $S$ is semiprime.

The quasi-ideal $\{O\} \subseteq\langle S Q\rangle \cap\langle Q S\rangle \subseteq Q$ satisfies either

$$
\langle S Q\rangle \cap\langle Q S\rangle=\{O\} \text { or }\langle S Q\rangle \cap\langle Q S\rangle=Q
$$

since $Q$ is $O$-minimal. To show $\langle S Q\rangle \cap\langle Q S\rangle=Q$ we go by contradiction and assume $\langle S Q\rangle \cap\langle Q S\rangle=$ $\{O\}$. Then $\langle Q S Q\rangle \subseteq\langle S Q\rangle \cap\langle Q S\rangle=\{O\}$ implies $\left\langle\langle S Q\rangle^{2}\right\rangle=\langle S Q S Q\rangle=\{O\}$, which together with $\langle S Q\rangle \neq\{O\}$ contradicts that $S$ is semiprime. So it remains to show that the one-sided ideals $\langle S Q\rangle$ and $\langle Q S\rangle$ of $S$ are $O$-minimal. Assume $\{O\} \subset L \subseteq\langle S Q\rangle$ for a left ideal $L$ of $S$. Then the quasi-ideal $\langle S L\rangle \cap\langle Q S\rangle$ satisfies either

$$
\langle S L\rangle \cap\langle Q S\rangle=\{O\} \quad \text { or }\langle S L\rangle \cap\langle Q S\rangle=Q,
$$

since $\{O\} \subseteq\langle S L\rangle \cap\langle Q S\rangle \subseteq\langle S Q\rangle \cap\langle Q S\rangle \subseteq Q$ holds and $Q$ is assumed to be $O$-minimal. Now $\langle Q L\rangle \subseteq\langle S L\rangle \cap\langle Q S\rangle=\{O\}$ would imply $\left\langle L^{2}\right\rangle \subseteq\langle\langle S Q\rangle L\rangle=\langle S Q L\rangle=\{O\}$, but $S$ is assumed to be semiprime. So we obtain $\langle S L\rangle \cap\langle Q S\rangle=Q$ which implies $Q \subseteq\langle S L\rangle \subseteq L$ and in turn $\langle S Q\rangle \subseteq\langle S L\rangle \subseteq L$, hence $L=\langle S Q\rangle$, so $\langle S Q\rangle$ is $O$-minimal. Dually, the right ideal $\langle Q S\rangle$ of $S$ is also $O$-minimal.

Let $S$ be a semiring or semigroup with absorbing element $O$ or a ring. Then $S$ is called quasireflexive iff all two-sided ideals $A, B$ of $S$ satisfy the implication $\langle A B\rangle \subseteq\{O\} \Rightarrow\langle B A\rangle \subseteq\{O\}$ or 
cquivalently $A B \subseteq\{O\} \Rightarrow B A \subseteq\{O\}$. Obviously each semiprime semiring, scmigroup or ring is also quasi-reflexive, but not conversely.

For rings and semigroups the following lemma is a special case of Prop. 2.1 and Cor. 2.3 in [i].

LEMMA 3.5. Let $S$ be a semiring or a scmigroup with absorbing element $O$ or a ring. Then the following conditions are equivalent:

a) All two-sided ideals $A, B$ of $S$ satisfy $A B \subseteq\{O\} \Rightarrow B A \subseteq\{O\}$.

b) All left ideals $A, B$ of $S$ satisfy $A B \subseteq\{O\} \Rightarrow B A \subseteq\{O\}$.

c) All right ideals $A, B$ of $S$ satisfy $A B \subseteq\{O\} \Rightarrow B A \subseteq\{O\}$.

PROOF. The implications (b) $\Rightarrow(\mathrm{a})$ and (c) $\Rightarrow$ (a) are obvious. For the proof of (a) $\Rightarrow(\mathrm{b})$, assume $A B \subseteq\{O\}$ for any left ideals $A, B$ of $S$. Then $\langle A \cup A S\rangle$ and $\langle B \cup B S\rangle$ are two-sided irlcals of $S$ satisfying

$$
\langle A \cup A S\rangle\langle B \cup B S\rangle \subseteq\langle A B \cup A B S \cup A S B \cup A S B S\rangle \subseteq\{O\}
$$

and hence by (a) also $\langle B \cup B S\rangle\langle A \cup A S\rangle \subseteq\{O\}$, which clearly implies $B A \subseteq\{O\}$. The implication (a) $\Rightarrow$ (c) is the dual of $(a) \Rightarrow(b)$.

LEMMA 3.6. Let $L$ be an (O-)minimal left ideal of a semiring, a ring or a semigroup $S$ and $O \neq e=e^{2} \in L$. Then $\left((e L)^{\prime}, \cdot\right)$ is a subgroup of $(S, \cdot)$.

PROOF. Clearly, $(e L, \cdot)$ is a subsemigroup of $(S, \cdot)$ with $e$ as left identity. In fact even $\left((e L)^{\prime}, \cdot\right)$ is a subsemigroup of $(S, \cdot)$, since the assumption $a b=O$ for any $a, b \in(e L)^{\prime}$ (together with $(a)_{l}=\langle a \cup S a\rangle=L$ by the (O-)minimality of $\left.L\right)$ would imply $L b=\langle a \cup S a\rangle b=\{O\}$ in contradiction to $O \neq b=e b \in e L$. To show that $\left((e L)^{\prime}, \cdot\right)$ is a group consider any element $e l \in(e L)^{\prime}$. Then $O \neq e l=e e l \in L e l$ and $\langle S(L e l)\rangle=\langle(S L) e l\rangle \subseteq\langle L e l\rangle=L e l$ shows that $\{O\} \neq L e l$ is a left ideal of $S$ which is clearly contained in $L$. This yields $L e l=L$ since $L$ is $(\mathrm{O}-)$ minimal. Now we conclude $e L e l=e L$ and so there exists an element $x=e k \in e L$ such that $x e l=e k e l=e e=e$ which shows that $\left((e L)^{\prime}, \cdot\right)$ is a group since $x=O$ is of course impossible.

For quasi-reflexive rings and semigroups the following proposition and theorem correspond to Props. 4.1 and 4.3 in [7]. They contain Thm. 7.4 of [3] for semiprime rings and semigroups as special cases.

THEOREM 3.7. Let $S$ be a semiring or semigroup with absorbing element $O$ or a ring such that $S$ is quasi-reflexive. Then for each element $e=e^{2} \neq O$ of $S$ the following statements are equivalent:

a) $S e$ is an $O$-minimal left ideal of $S$.

$\left.\mathrm{a}^{\prime}\right) e S$ is an $O$-minimal right ideal of $S$.

b) $e S e$ is an $O$-minimal quasi-ideal of $S$.

c) $\left((e S e)^{\prime}, \cdot\right)$ is a subgroup of $(S, \cdot)$.

PROOF. The equivalence (b) $\Leftrightarrow$ (c) is known for arbitrary semirings ([8], Satz 4.5), arbitrary rings ([3], Prop. 6.11) and arbitrary semigroups ([3], Prop. 5.6). From Lemma 3.6 and its dual we obtain $(\mathrm{a}) \Rightarrow(\mathrm{c})$ and $\left(\mathrm{a}^{\prime}\right) \Rightarrow(\mathrm{c})$. For $(\mathrm{b}) \Rightarrow$ (a) let $\{O\} \neq L \subseteq S e$ be a left ideal of $S$. Then $L e=L$ holds. Furthermore, $e L$ is a quasi-ideal of $S$ by Lemma 1.3 and we show $e L \neq\{O\}$ by contradiction. Indeed, $e L=\{O\}$ would imply $S e L=\{O\}$ and hence $L S e=\{O\}$ since $S$ is assumed to be quasi-reflexive. The latter would imply $L e=\{O\}$, contradicting $L e=L \neq\{O\}$. So we have $\{O\} \neq e L=e L e \subseteq e S e$, hence $e L=e S e$ since $e S e$ is assumed to be (O-)minimal. Of 
coursc $O \neq e$ is cancellable in the group $\left(\left(c S_{c}\right)^{\prime}, \cdot\right)$, so we conclude $L=S e$, hence the left ideal $S e$ is $(\mathrm{O}-)$ minimal. Similarly one shows $(b) \Rightarrow\left(a^{\prime}\right)$.

THEOREM 3.8. Let $S$ be a semiring or semigroup with absorbing element $O$ or a ring such that $S$ is quasi-reflexive. Then each $O$-minimal quasi-ideal $Q$ of $S$ satisfies either $Q^{2}=\{O\}$ or it is a canonical quasi-ideal of $S$.

PROOF. Let $Q$ be an $O$-minimal quasi-ideal of $S$ satisfying $Q^{2} \neq\{O\}$. Then by Thenrem 3.2 c) there exists an element $O \neq c=e^{2} \in S$ satisfying $Q=S e \cap \epsilon S=c S e$. Since $S$ is quasireflexive and $Q=e S e$ is assumed to be $O$-minimal, by Theorem 3.7 the one-sided idcals $S c$ and $e S$ of $S$ are also $O$-minimal, hence $Q$ is canonical.

Finally, we give some statements on canonical quasi-ideals for arbitrary scmirings, semigroups and rings. In the last both cases the following theorem and lemma correspond to Thm. 0.7 a), Thm. 6.7 b) and Prop. 6.9 in [3].

THEOREM 3.9. Let $S$ be a semiring, a ring or a semigroup and let $L$ and $R$ be ( $O$-)minimal left and right ideals of $S$. Then $\langle R L\rangle$ is either $\{O\}$ or a canonical quasi-ideal of $S$ satisfying $\langle R L\rangle=L \cap R$.

PROOF. We assume $\langle R L\rangle \neq\{O\}$, which clearly holds if $S$ has no absorbing element, and the same applies to statements like $"\langle S R L\rangle \neq\{O\} "$ or $" O \neq x$ " appearing in the following. By Lemma $1.2 \mathrm{c}$ ) and Theorem 3.1, we have $\langle R L\rangle \subseteq L \cap R=Q$ where $Q$ is a canonical quasi-ideal, and hence an $(O-)$ minimal quasi-ideal of $S$. So we have to show that $\langle R L\rangle$ is a quasi-ideal of $S$, that is $\langle S R L\rangle \cap\langle R L S\rangle \subseteq\langle R L\rangle$. This is trivial if $\langle S R L\rangle=\{O\}$ or $\langle R L S\rangle=\{O\}$, so let us assume $\langle S R L\rangle \neq\{O\}$ and $\langle R L S\rangle \neq\{O\}$. By the $(O$-)minimality of $L$ and $R$ we get $\langle S R L\rangle=L$ and $\langle R L S\rangle=R$. Clearly, $\langle S R L\rangle \neq\{O\}$ implies the existence of $O \neq x \in R L \subseteq L \cap R$ with $S x \neq\{O\}$. Since $L$ is $(O$-)minimal, we get $S x=\langle S x\rangle=L$ and $S x S=L S$. Now $R=\langle R L S\rangle \neq\{O\}$ implies $\{O\} \neq L S=S x S$, thus $\{O\} \neq x S=\langle x S\rangle \subseteq R$. Since $R$ is $(O-)$ minimal, this yields $x S=R$. Hence we can conclude $O \neq x \in R L=x S S x \subseteq x S x$, thus $x$ is regular in $S$. So we can apply Thm. 2.2 to $(x)_{l}=L$ and $(x)_{r}=R$ and we obtain $\langle R L\rangle=\left\langle(x)_{r}(x)_{l}\right\rangle=(x)_{l} \cap(x)_{r}=S x \cap x S=L \cap R$, which proves that $\langle R L)$ is a quasi-ideal of $S$ also in this case.

LEMMA 3.10. Let $S$ be a semiring, a ring or a semigroup and let $L$ be an $(O$-)minimal left ideal of $S$ and $a \in S$. Then $L a$ is an (O-)minimal left ideal of $S$ or $L a=\{O\}$ holds.

PROOF. For each left ideal $L$ of $S$ and $a \in S$ clearly $L a=\langle L a\rangle$ is a left ideal of $S$. Now assume $\{O\} \subseteq A \subseteq L a$ for a left ideal $A$ of $S$ and define $B$ by $\{b \in L \mid b a \in A\}$. Then $A=B a$ holds and $B$ is obviously a left ideal of $S$. From $\{O\} \subseteq B \subseteq L$ we ol, tain either $B=\{O\}$ or $B=L$, i.e. $A=\{O\}$ or $A=L a$. Hence $L a$ is an (O-)minimal left ideal of $S$.

Now we prove two theorems which contain Thms. 2.2 and 2.8 in [4] for rings and Thms. 2.3 and 2.9 in [5] for semigroups.

THEOREM 3.11. Let $S$ be a semiring, a ring or a semigroup and $Q$ a canoni-cal quasi-ideal of $S$. Hence there is a (unique) (O-)minimal left ideal $L$ and a (unique) $(O$-)minimal right ideal $R$ of $S$ satisfying $Q=L \cap \dot{R}$. Then for each $a \in S$ either $Q a=\langle Q a\rangle$ is a canonical quasi-ideal of $S$ satisfying $Q a=L a \cap R$ or $Q a=\{O\}$ holds.

PFOOF. We assume $Q a \neq\{O\}$. Then obviously $Q a \subseteq L a \cap R a \subseteq L a \cap R=K$ holds. Since $L a$ is an (O-)minimal left ideal of $S$ by Lemma $3.10, K$ is a canonical quasi-ideal of $S$. We are now 
going to show $L a \cap R=K \subseteq Q a$. Consider some $k \neq O$ of $K$. Then $k=b a$ for some $b \in L$ yiclds $R=(k)_{r} \subseteq(b)_{r}$. Since $Q$ is contained in the $(O$-)minimal left ideal $L$, each $q \neq O$ of $Q$ satisfics $S q=L$ or $S q=\{O\}$. In the last case, $L=(q) \subseteq \subseteq Q$ implics $L=Q$, which makes $K=L a \cap R \subseteq(Q a$ trivial. Otherwise, there is at least one $q \neq O$ of $Q$ such that $S q=L$ holds, which yiclds $s q=b$ for the $b$ considered above and some $s \in S$. This and $q \in Q \subseteq R$ imply $R \subseteq(b)_{r}=(s q)_{r} \subseteq . R$. By the dual of Lemma 3.10, $s R$ is an (O-)minimal right ideal of $S$ which yields $R=s R$ and hence $b \in R$. So we have obtained $b \in L \cap R=Q$ and thus $k=b a \in Q a$, i.e. again $K=L a \cap R \subseteq Q a$. From $Q a \subseteq K$ as stated above we obtain $Q a=L a \cap R$.

THEOREM 3.12. Let $S$ be a semiring, a ring or a semigroup and let $Q_{1}, Q_{2}$ be canonical quasi-ideals of $S$. Then $\left\langle Q_{1} Q_{2}\right\rangle$ is a canonical quasi-ideal of $S$ or $\left\langle Q_{1} Q_{2}\right\rangle=\{O\}$ holds.

PROOF. Since $Q_{1}, Q_{2}$ are canonical quasi-ideals of $S$, there are $\left(O\right.$-)minimal left ideals $L_{1}, L_{2}$ and (O-)minimal right ideals $R_{1}, R_{2}$ of $S$ such that $Q_{1}=L_{1} \cap R_{1}$ and $Q_{2}=L_{2} \cap R_{2}$. Let us assume $\left\langle Q_{1} Q_{2}\right\rangle \neq\{O\}$. Then

$$
\left\langle Q_{1} Q_{2}\right\rangle=\left\langle\left(L_{1} \cap R_{1}\right)\left(L_{2} \cap R_{2}\right)\right\rangle \subseteq\left\langle R_{1} L_{2}\right\rangle \subseteq L_{2} \cap R_{1}
$$

Because of $\left\langle Q_{1} Q_{2}\right\rangle \neq\{O\}$ there is a $O \neq q_{2} \in Q_{2}$ with $Q_{1} q_{2} \neq\{O\}$. Then Theorem 3.11 implies $Q_{1} q_{2}=L_{1} q_{2} \cap R_{1}$. From $\{O\} \neq Q_{1} q_{2} \subseteq L_{1} q_{2} \subseteq L_{1} L_{2} \subseteq L_{2}$ we get $L_{1} q_{2}=L_{2}$ since $L_{2}$ is $(O$ )minimal, so we have $Q_{1} q_{2}=L_{2} \cap R_{1}$. Now $L_{2} \cap R_{1}=Q_{1} q_{2} \subseteq Q_{1} Q_{2} \subseteq\left\langle Q_{1} Q_{2}\right\rangle \subseteq L_{2} \cap R_{1}$ implies $\left\langle Q_{1} Q_{2}\right\rangle=L_{2} \cap R_{1}$, hence $\left\langle Q_{1} Q_{2}\right\rangle$ is a canonical quasi-ideal of $S$.

The last corollary is an immediate consequence of (1.6) and Theorem 3.12.

COROLLARY 3.13. Let $S$ be a semiring, a ring or a semigroup which contains at least one canonical quasi-ideal and let $\mathcal{V}$ denote the set of all canonical quasi-ideals of $S$. Then $\mathcal{V}$ or $\mathcal{V} \cup\{O\}$ is a semigroup with respect to the "product" $\left\langle Q_{1} Q_{2}\right\rangle$, which has $\{O\}$ as absorbing element in the latter case.

\section{REFERENCES}

[1] CLIFFORD,A.H. Remarks on 0-minimal quasi-ideals in semigroups, Semigroup Forum, 16 (1978), 183-196.

[2] GLUSKIN,L.M. and STEINFELD,O. Rings (semigroups) coutaining minimal (0-minimal) right and left ideals, Publ. Math. Debr., 25 (1978), 275-280.

[3] STEINFELD,O. Quasi-ideals in rings and semigroups, Akademiai Kiado, Budapest, 1978.

[4] STEINFELD,O. On canonical quasi-ideals in rings, Annales Univ. Sci. Budapest. R. Eötvös, Sectio Math. 31 (1988), 171-178.

[5] STEINFELD,O. and THANG,T.T. Remarks on canonical quasi-ideals in semigroups,Beitr. Alg. Geom. 26 (1987), 127-135.

[6] VAN ROOYEN,G.W.S. On quasi-reflexive Rings (Semigroups), Proc. Japan Acad., 64, Ser. A, No.9 (1988), 337-340.

[7] VAN ROOYEN,G.W.S. and WEINERT,H.J. On quasi-reflexive rings and semigroups, Quaestiones Mathematicae 14 (1991), 361-369.

[8] WEINERT,H.J. Über Quasiideale in Halbringen, Contributions to General Algebra 2,Proceedings of the Klagenfurt Conference, June 10-13, 1982. Verlag Hölder-Pichler-Tempsky, Wien 1983 - Verlag B.G. Teubner, Stuttgart, 375-394.

[9] WEINERT,H.J. On quasi-ideals in rings, Acta Math. Acad. Sci. Hung. 43 (1984), 85-92.

[10] WEINERT,H.J. Generalized Semialgebras over Semirings, Proceedings of a Conference on Semigroups, Theory and Applications, Oberwolfach, Germany, 1986, Lecture Notes in Mathematics, Band 1320 (1988), 380-416. 


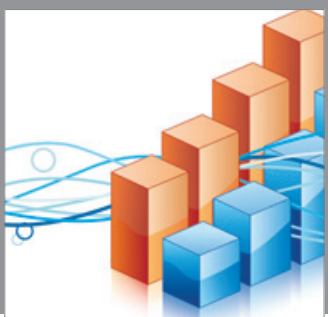

Advances in

Operations Research

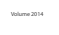

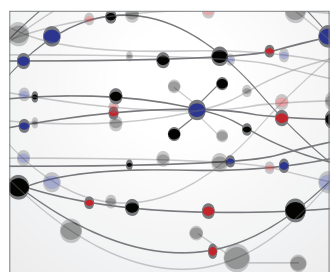

\section{The Scientific} World Journal
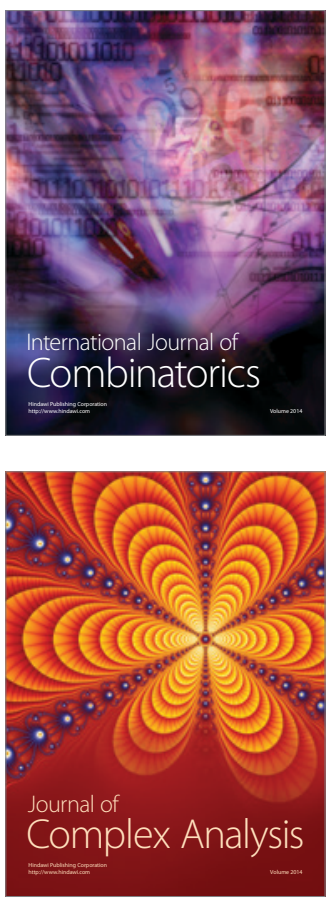

International Journal of

Mathematics and

Mathematical

Sciences
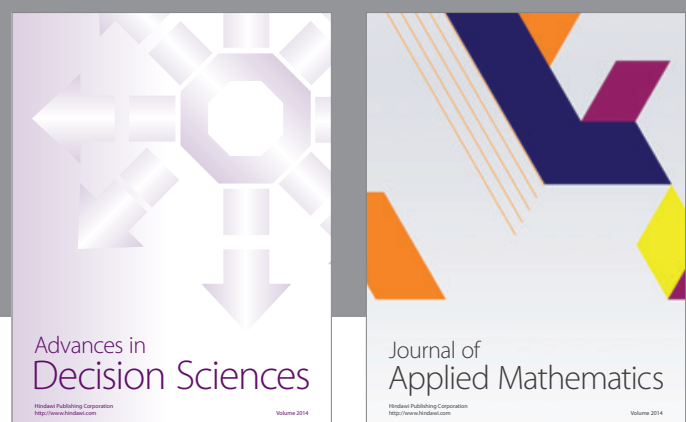

Journal of

Applied Mathematics
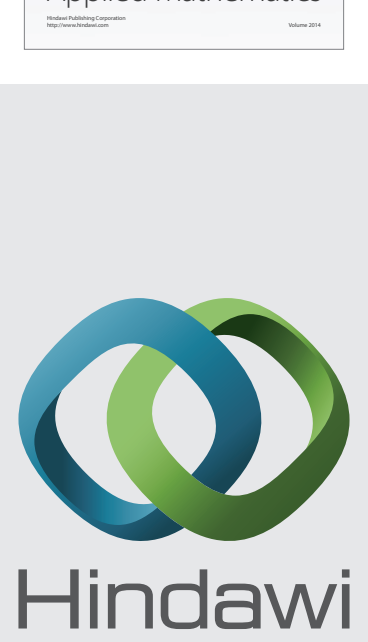

Submit your manuscripts at http://www.hindawi.com
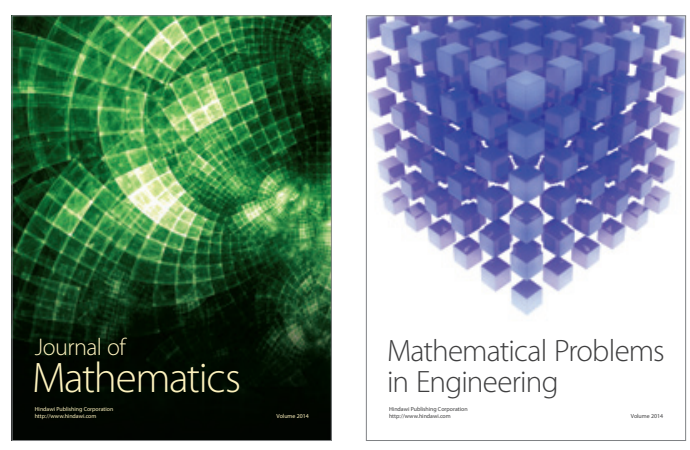

Mathematical Problems in Engineering
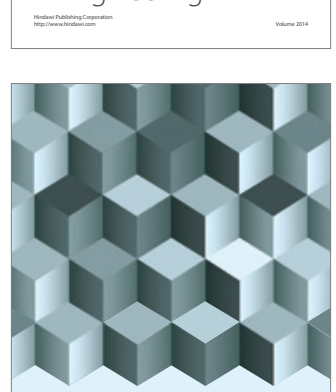

Journal of

Function Spaces
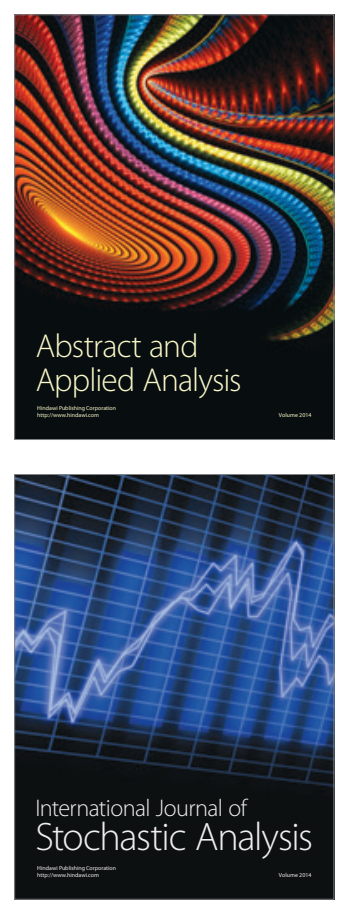

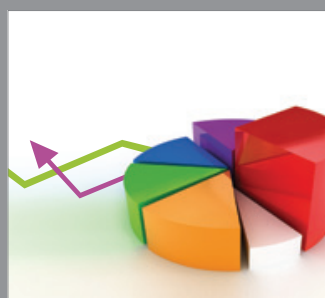

ournal of

Probability and Statistics

Promensencen
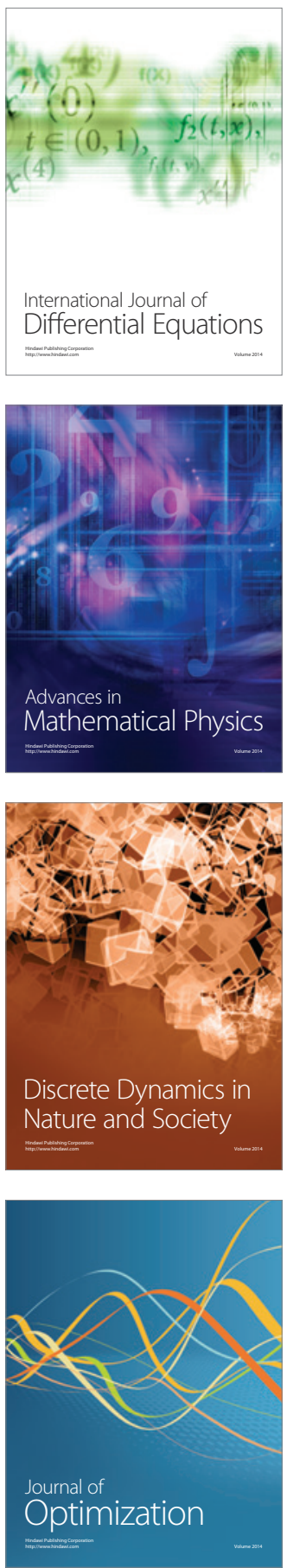